\title{
Olanzapine was more effective than lorazepam at 2 hours but not at 24 hours in bipolar mania with acute agitation
}

Meehan K, Zhang F, David S, et al. A double-blind, randomized comparison of the efficacy and safety of intramuscular
injections of olanzapine, lorazepam, or placebo in treating acutely agitated patients diagnosed with bipolar mania.J Clin
Psychopharmacol 2001 Aug;21:389-97.

QUESTION: In patients with bipolar mania who are acutely agitated, how effective are olanzapine, lorazepam, and placebo for rapidly calming patients?

Design

Randomised (unclear allocation concealment*), blinded (unclear)*, placebo-controlled trial.

Setting

USA and Romania.

\section{Patients}

201 patients $\geqslant 18$ years of age (mean age 40 y, 53\% men) who had bipolar disorder, mania, or a mixed diagnosis confirmed with the Structured Clinical Interview for DSMIII- $R$ considered by the site physician to have severe agitation requiring injections and who had a total score $\geqslant 14$ on the 5 item Positive and Negative Syndrome Scale-Excited Component (PANSS-EC) scale with $\geqslant 1$ individual score of $\geqslant 4$. Two patients were not included in the analysis.

Source of funding: Eli Lilly and Company.

For correspondence: Dr A Breier, Lilly

Research Laboratories, Eli Lilly and Company, Lilly Corporate Center, Indianapolis, $I N$ 46285, USA.

Breier_alan@lilly.com.

\section{Intervention}

Patients were allocated to 1 of 3 groups. Patients received 1-3 intramuscular injections. First and second injections $\geqslant 2$ hours apart were olanzapine ( $\mathrm{n}=99), 10 \mathrm{mg}$ each; lorazepam $(n=51), 2 \mathrm{mg}$ each; and placebo $(\mathrm{n}=51)$. Third injections $\geqslant 1$ hour after the second were olanzapine, 5 mg; lorazepam, $1 \mathrm{mg}$; and olanzapine (placebo group), 10 mg. All were given $<20$ hours after the first injection.

\section{Main outcome measures}

Agitation (PANSS-EC scale; Agitated Behavior Scale [ABS] total score; Agitation-Calmness Evaluation Scale [ACES]) at 2 and 24 hours after the first injection.

\section{Main results}

Analysis was by intention to treat with last observation carried forward. Olanzapine decreased agitation more than lorazepam at 2 hours $(\mathrm{p} \leqslant 0.006)$, but groups did not differ at 24 hours $(\mathrm{p} \geqslant 0.8)$ (table). Olanzapine decreased agitation more than placebo at 2 and 24 hours $(p \leqslant 0.025)$ (table). A greater change in ABS and ACES scores (decreased agitation) occurred in the lorazepam group than in placebo at 2 and 24 hours $(p \leqslant 0.01)$, but groups had a similar change in PANSS-EC score $(\mathrm{p} \geqslant 0.05)$ (table).

\section{Conclusions}

Olanzapine was more effective than lorazepam at 2 hours, but was as effective at 24 hours for rapidly calming patients with bipolar mania who were acutely agitated. Olanzapine was more effective than placebo. Lorazepam was more effective than placebo on 2 of 3 agitation scales.

*See glossary.

Olanzapine (Olan) v lorazepam (Lor) v placebo (Pl) in bipolar mania with acute agitation $\dagger$

\begin{tabular}{|c|c|c|c|c|c|c|}
\hline \multirow[b]{2}{*}{ Outcomes } & \multicolumn{6}{|c|}{ Difference in mean score change from baseline $(95 \% \mathrm{Cl}) \neq$} \\
\hline & Olan $v$ Lor $(2 \mathrm{~h})$ & Olan $v$ Lor $(24 \mathrm{~h})$ & Olan v PI (2 h) & Olan v PI (24 h) & Lor $v \mathrm{PI}(\mathbf{2} \mathrm{h})$ & Lor v PI (24 h) \\
\hline Decrease in PANSS-EC score & 2.85 (1.18 to 4.52$)$ & $0.13(-1.54$ to 1.80$)$ & 4.76 (3.14 to 6.38$)$ & $1.84(0.26$ to 3.42$)$ & $1.91(-0.04$ to 3.86$)$ & $1.71(-0.18$ to 3.60$)$ \\
\hline Decrease in ABS score & 2.91 (0.81 to 5.02$)$ & $0.12(-1.93$ to 2.17$)$ & $6.52(4.50$ to 8.54$)$ & $3.16(1.18$ to 5.14$)$ & $3.61(1.27$ to 5.95$)$ & $3.04(0.86$ to 5.22$)$ \\
\hline Increase in ACES score & $1.02(0.41$ to 1.63$)$ & $0.02(-0.26$ to 0.30$)$ & 2.08 (1.50 to 2.66$)$ & $0.48(0.17$ to 0.79$)$ & $1.06(0.43$ to 1.69$)$ & $0.50(0.15$ to 0.85$)$ \\
\hline
\end{tabular}

+PANSS-EC=Positive and Negative Syndrome Scale-Excited Component (decrease=decreased agitation); ABS=Agitated Behaviour Scale (decrease=decreased agitation); $A C E S=$ Agitation-Calmness Evaluation Scale (increase=decreased agitation). $¥ \mathrm{Cl}$ calculated from data in article.

\section{COMMENTARY}

The study by Meehan $e t$ al looks at the role of olanzapine in the resolution of acute agitation in bipolar mania and therefore strays into a number of grey areas. The introduction of acute injectable atypical antipsychotics has been eagerly awaited in several quarters-somewhat wrongly in my view. The therapeutic aim in acute agitation is sedation rather than an unattainable instant antipsychotic effect, while the inevitable spontaneous remission sets in, possibly aided at the outset with antimanic agents like lithium or valproate. Therefore, the role of an atypical antipsychotic in these patients is without hypothetical foundation.

I believe also that the participants in this study cannot be typical of real world patients. They have given written informed consent and some were successfully randomised to placebo.

Whatever the validity of the PANSS-EC, ABS, and the ACES, these must be mildly ill patients and not the target population for intramuscular intervention. I cannot imagine any of my acutely agitated manic patients who have been admitted to my psychiatric intensive care unit being able to participate in this trial.

There are some general clinical trial issues here. Good evidence on the treatment of acutely disturbed patients is missing because of the perceived difficulty in performing trials in non-consenting patients. However, close reading of the recent draft of the Helsinki declaration, the European directive on clinical trials, and the Nuffield council documents on bioethics reveals that trials in non-consenting patients are permitted on 2 conditions: (1) no other context exists in which to answer the question, and (2) all trial participants get clear therapeutic benefit from whichever arm they are randomised to. So placebos cannot be used in these studies. More importantly, this extreme end of psychiatric practice where randomised controlled trials are difficult is an example where observational and naturalistic studies can guide practice and utility.

Clearly, Meehan et al's study is important for labelling purposes, but the real proof will come from real world, phase IV studies. Robert Kerwin, PhD, MB, BChir, DSc, FRCPsych Institute of Psychiatry London, UK 